\title{
Quelques aspects de la prévision des mouvements de terrain
}

C. AZIMI

P. DESVARREUX

Association pour le Développement des Recherches sur les Glissements de Terrains (ADRGT), BP 17 . 38610 Gières
Les mouvements de terrain au sens large (glissements de terrains meubles, mouvements au sein de formations rocheuses) posent deux problèmes liés à la prévision: - la prévision de leur propagation dans l'espace; - la prévision de leur évolution dans le temps. On ne s'intéresse ici qu'au second type de prévision en distinguant les phénomènes dans lesquels la vitesse dépend des conditions hydrauliques et ceux dans lesquelles elle n'en dépend pas.

On décrit quatre cas sur lesquels des prévisions de comportement ont été tentées, parfois avec succès, parfois sans. Deux de ces cas concernent des matériaux meubles et deux des matériaux rocheux. On indique les critères pour déceler le passage des mouvements à vitesses "réversibles » aux mouvements à vitesses irréversibles.

\section{Some aspects of forecasting the behaviour of slope movements}

Slope movements (i.e. landslides, rock slides, rockfalis) induce two kinds of problems, related to forecasting:

- forecasting the maximum extent of rapid movements:

- forecasting time - dependent behaviour of movements,

This paper is concerned only with the second type of forecasting.

The authors make a distinction between the movements in which velocity depends on pore pressure conditions, and those in which velocity is not dependent on these conditions. The authors describe four cases of prediction studies, with successful or unsuccessful results in behaviour forecasting. Two of these examples are concerned with soils and two ones with rocks. Criteria are given for detecting the changes in behaviour. that lead to non reservible movements.
} 


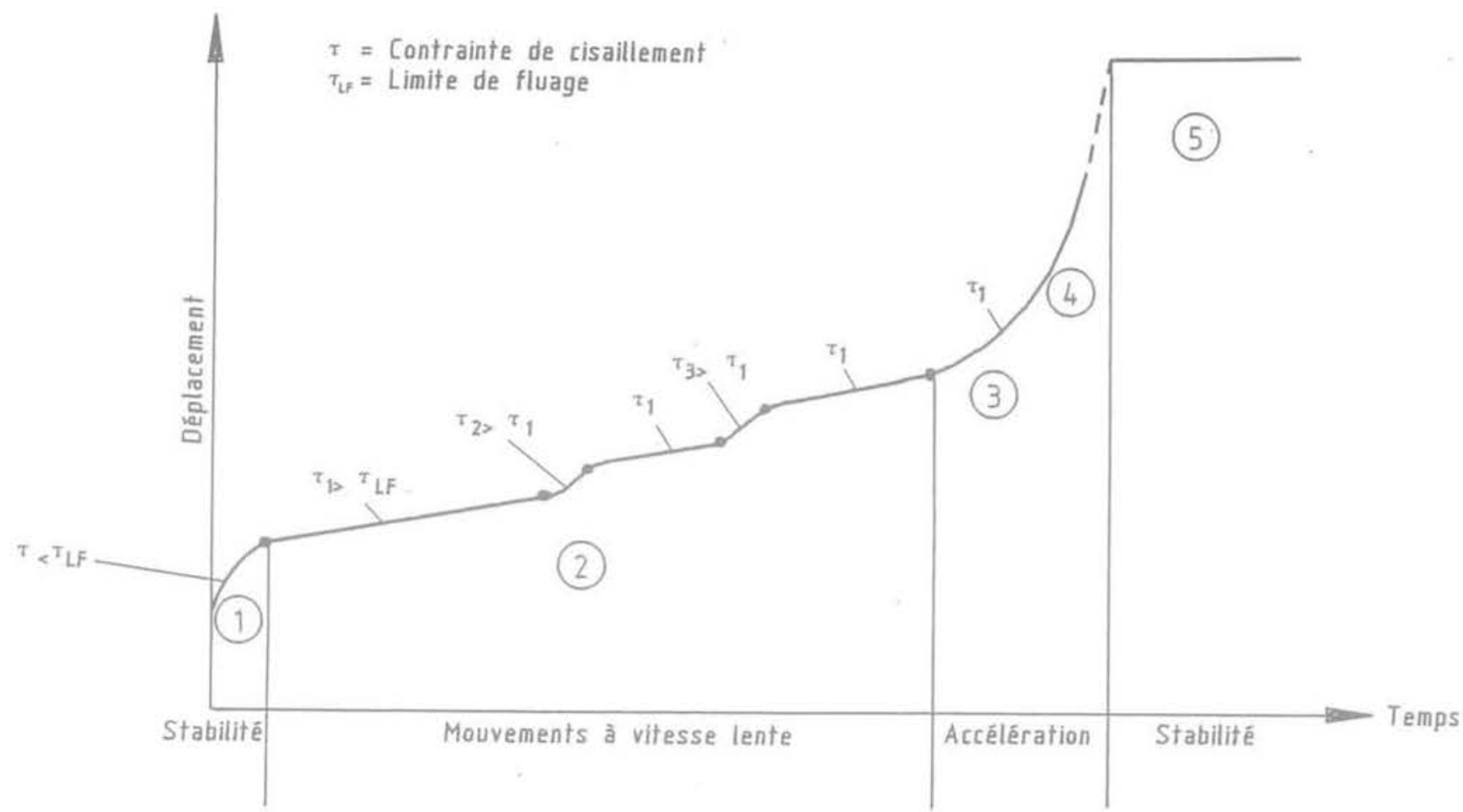

Eboulement

FiQ. Différents états d'un mouvement de terrain. Different stages of slope movements.

\section{1}

\section{Introduction - particularités cinématiques des mouvements du sol}

Un mouvement de terrain est un phénomène géologique qui évolue dans le temps. Quel que soit le matériau concerné (sol ou roche), on peut distinguer trois états, sur le plan cinématique (Fig. 1), qu'on peut rapprocher, par analogie, des divers stades de fluage mis en évidence sur échantillons ${ }^{\text {(1) }}$.

L'état (1) correspond à la stabilité: toute nouvelle sollicitation provoque alors une déformation instantanée. Il peut y avoir également une déformation différée mais celle-ci ralentit très vite ( fluage » primaire).

L'état (2) correspond à des mouvements à vitesse pratiquement constante, en général faible (quelques $\mathrm{mm}$ à quelques $\mathrm{cm} / \mathrm{an}$ ) qui peuvent durer très longtemps (10 à 50 ans) ou moins ( 2 à 3 ans) dans le cas de certains éboulements. Si la contrainte varie (par exemple variation de pression interstitielle), la vitesse de déplacement varie aussi, mais on reste dans le même type de comportement, qui est celui du «fluage» secondaire. En particulier si les contraintes diminuent en deçà de la "limite de fluage », les mouvements s'arrêtent. En ce sens, tant qu'on est dans ce type de comportement, on peut considérer la vitesse des mouvements de terrains comme un phénomène réversible.

L'état (3) correspond à une accélération continue sous contrainte constante menant à la rupture (4). On atteint de très grandes vitesses et on peut avoir de grands déplacements. Cette accélération est très différente de celles qui peuvent se produire en phase (2). Elle correspond au «fluage tertiaire », lequel représente l'évolution ultime du fluage secondaire, la contrainte restant constante. Dans ce type de comportement, on considère les mouvements comme irréversibles.

Au cours des phases (3) et (4) les matériaux subissent une redistribution des masses qui conduit à un nouvel état de stabilité (?).

Compte tenu de ce qui précède, on doit distinguer deux types très différents de phénomènes de mouvements de terrains:

- ceux dont la vitesse dépend des conditions hydrauliques ${ }^{(2)}$;

- ceux dont la vitesse ne dépend pas des conditions hydrauliques.

Dans la suite nous allons illustrer par quelques exemples pris dans chacun des deux types de phénomènes comment on a pu chercher à répondre à la question de prévision du comportement.

En effet, devant un phénomène de mouvement de terrain, si on est confronté à des impératifs de gestion de la sécurité, les deux questions capitales auxquelles on doit s'efforcer de répondre sont:

- comment le phénomène peut-il évoluer dans le temps? (prévision temporelle);

- comment le phénomène peut-il s'étendre spatialement? (prèvision spatiale).

Dans le présent article on ne s'intéressera qu'au premier type de prévision et on va présenter quatre cas sur lesquels des prévisions de comportement ont été tentées avec plus ou moins de succès. Ces cas ont été choisis car ils concernent aussi bien des matériaux meubles que rocheux, avec des particularités diverses quant à la cinématique, pour tenter d'illustrer la grande variété des problèmes qui se posent (Fig. 2).

(1) Pour cette raison nous parlons ici de «fluage n.

(2) On exclut du sujet de cet article les mouvements liés à des sollicita. tions dynamiques rapides (séisme). 


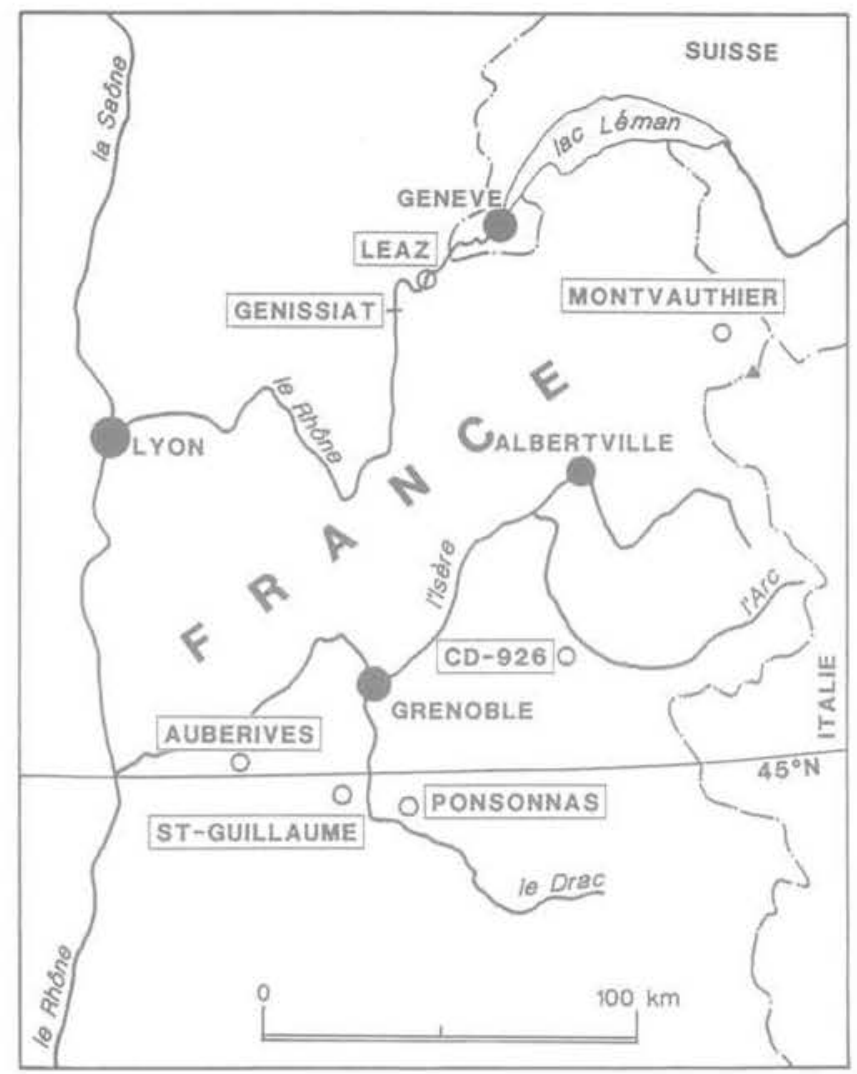

Fig 2 Situation des cas présentés. General map showing the location.

\section{2}

\section{Exemple du glissement de Leaz (Ain)}

\section{Localisation - Circonstances}

Ce glissement de terrain naturel domine la retenue de Génissiat sur le Rhône. II était connu dès 1934 avant établissement de la retenue, mais n'avait pas fait l'objet d'études particulières. A partir de 1964, suite à une réactivation des mouvements, une surveillance a été progressivement mise en place, parallèlement avec des reconnaissances géologiques classiques. Le but de ces études était de préciser le volume en mouvement, le rôle éventuel de la retenue dans ces mouvements, de prévoir dans quelles conditions des masses importantes de matériaux pourraient arriver dans la retenue et à quelles vitesses (ceci dans le but d'apprécier les conséquences du phénomène et en particulier les caractéristiques de l'onde hydraulique engendrée).

\section{Description du site}

La synthèse des reconnaissances géologiques est présentée en figure 3 et on peut retenir les points suivants:

- le substratum de marnes situé à 30-40 m de profondeur est stable, de mème qu'une terrasse d'alluvions anciennes (aucun mouvement entre 1965 et 1993). La retenue n'a donc aucune influence sur le glissement; - le glissement s'effectue au sein d'une formation d'argiles litées d'origine glacio-lacustre, très répandue dans la région et caractérisé par:

$$
\begin{aligned}
& \mathrm{W}_{\mathrm{L}}=35-50 \% \\
& \mathrm{IP}^{2}=20-30 \%
\end{aligned}
$$$$
\left.\phi \mathrm{r}=19,5^{\circ} \mathrm{c}^{\prime}=0 \text { (cisaillement à } 1 \mu / \mathrm{mn}\right) ;
$$

- le volume des matériaux en mouvement est de $1,4.10^{6} \mathrm{~m}^{3}$, caractérisés en 1969 par des vitesses de 5$10 \mathrm{~cm} / a n$ et de $10-30 \mathrm{~cm} / \mathrm{an}$ dans la zone la plus active représentant $100000 \mathrm{~m}^{3}$ (vitesses maximales de l'ordre de $1 \mathrm{~m} /$ an pour certains points).

\section{3}

\section{Surveillance et résultats}

Les mesures de déplacement ont commencé en 1964 mais depuis 1977 le système de surveillance comporte:

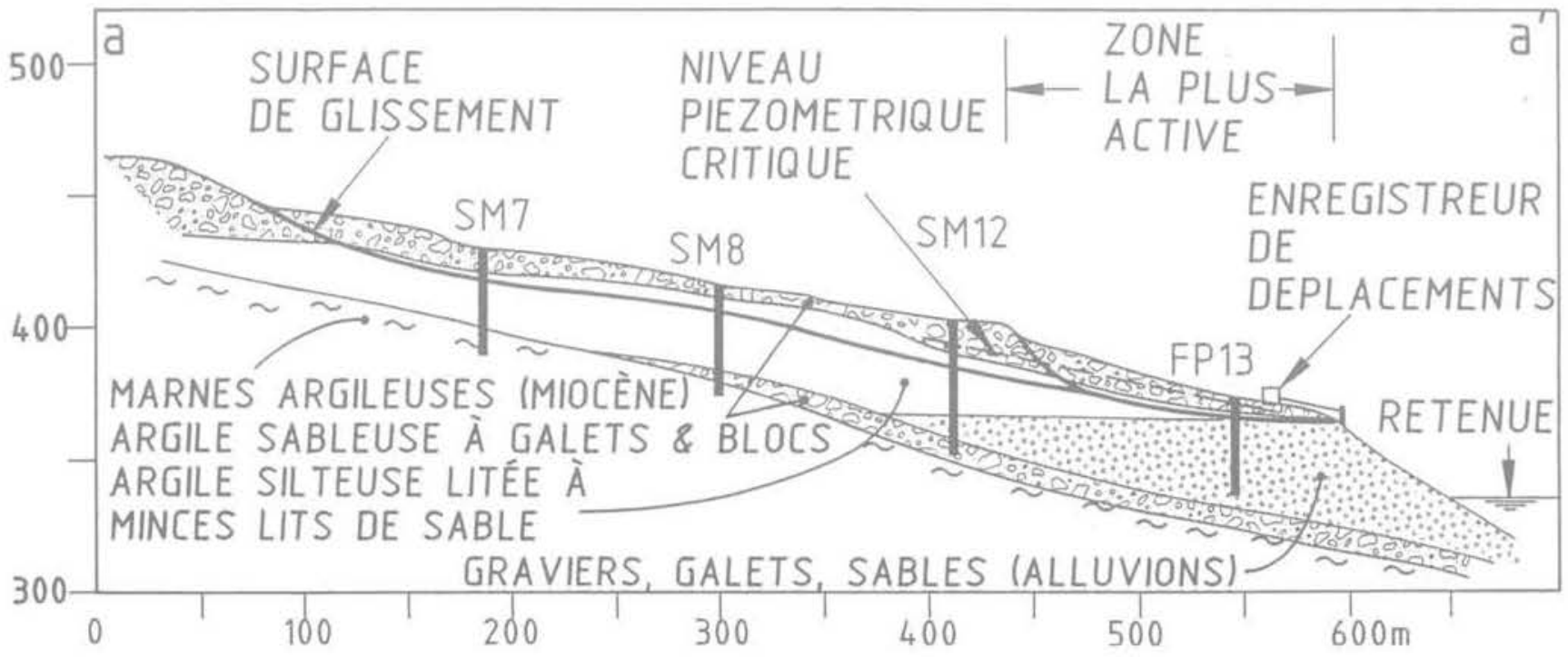

FIG. 3 Glissement de Leaz (Ain) - Coupe géologique schématique.

Leaz landslide - Simplified geological section. 
- des mesures annuelles en triangulation sur 25 témoins :

- des mesures mensuelles au distancemètre sur 11 témoins répartis dans le glissement:

- un enregistrement en continu des déplacements d'un point de la zone la plus active:

- un enregistrement des niveaux d'eau dans 4 piézomètres et des mesures mensuelles dans 8 autres;

- un enregistrement de la pluviométrie.

Sur la figure 4, on a représenté un exemple de 10 mois d'enregistrement corrélés avec des variations du niveau piézométrique à proximité de l'enregistreur.

On a ainsi pu mettre en évidence trois points fondamentaux:

- les périodes d'activité sont réduites dans le temps alors qu'on a plusieurs mois " (d'arrêt») par an des mouvements (à 1 ou $2 \mathrm{~mm}$ près), correspondant à l'état (1) de stabilité ; - l'activité du glissement se produit lorsque le niveau d'eau dépasse une valeur critique $\mathrm{N}_{0}$ :

- en phase d'activité, on peut lier la vitesse instantanée des déplacements au niveau d'eau par une formule approchée du type $\mathrm{V}=\mathrm{k}(\mathrm{N}-\mathrm{N})$. On est alors dans l'état (2) des mouvements réversibles (Fig. 1).

On a donc pu proposer un certain schéma de comportement du glissement qui permettrait de rendre compte des vitesses observées en fonction des niveaux d'eau dans le terrain.

Ce schéma comporte un modèle hydraulique permettant à partir de la pluviométrie journalière de déterminer le niveau piézométrique au sondage FP13, et un modèle mécanique permettant de relier ce niveau piézométrique à la vitesse instantanée. La figure 5 indique

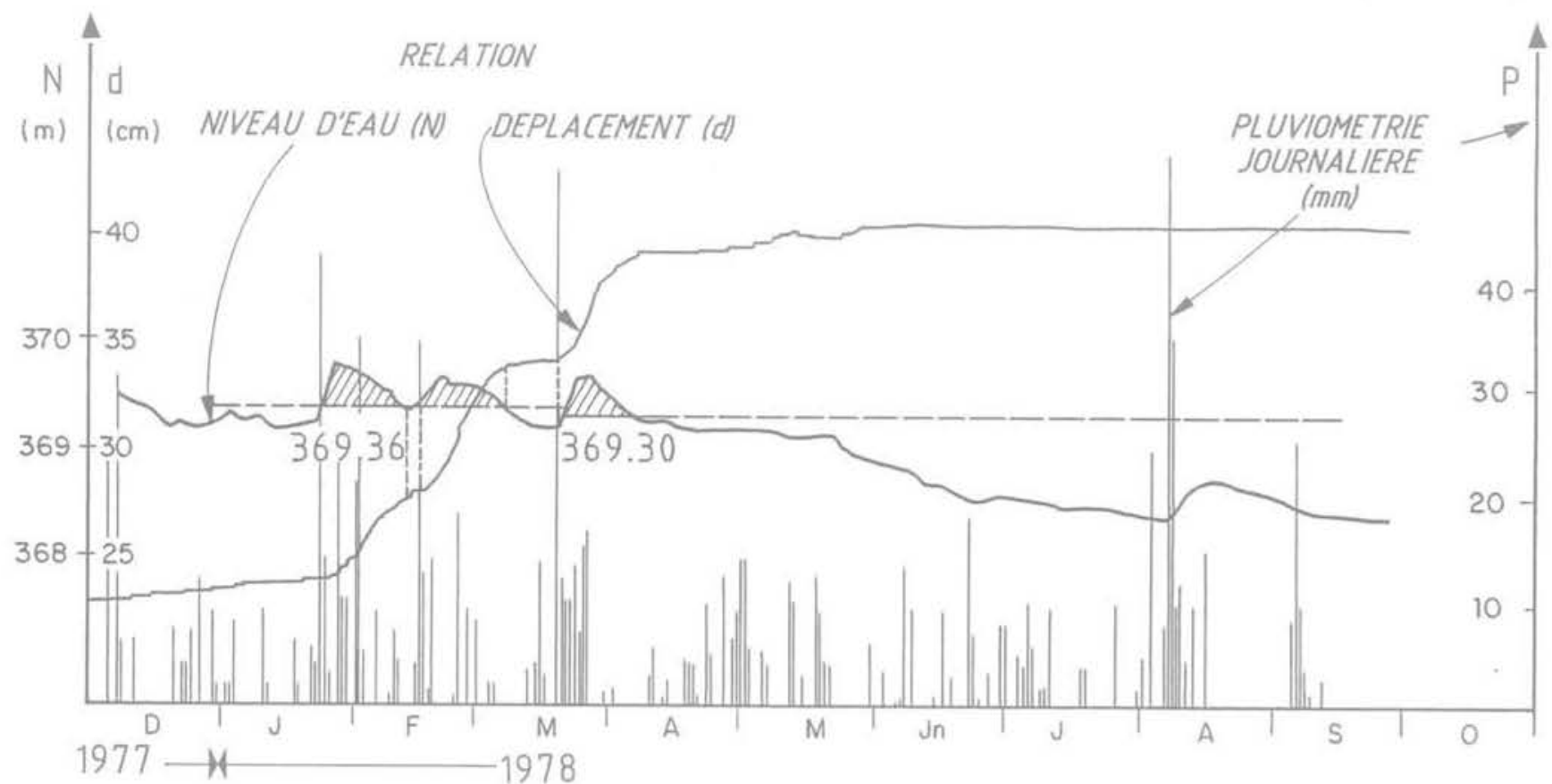

Flo.4 Glissement de Leaz - Enregistrements simultanés de piézométrie et déplacements. Leaz landslide - Piezometric level and displacement recordings.

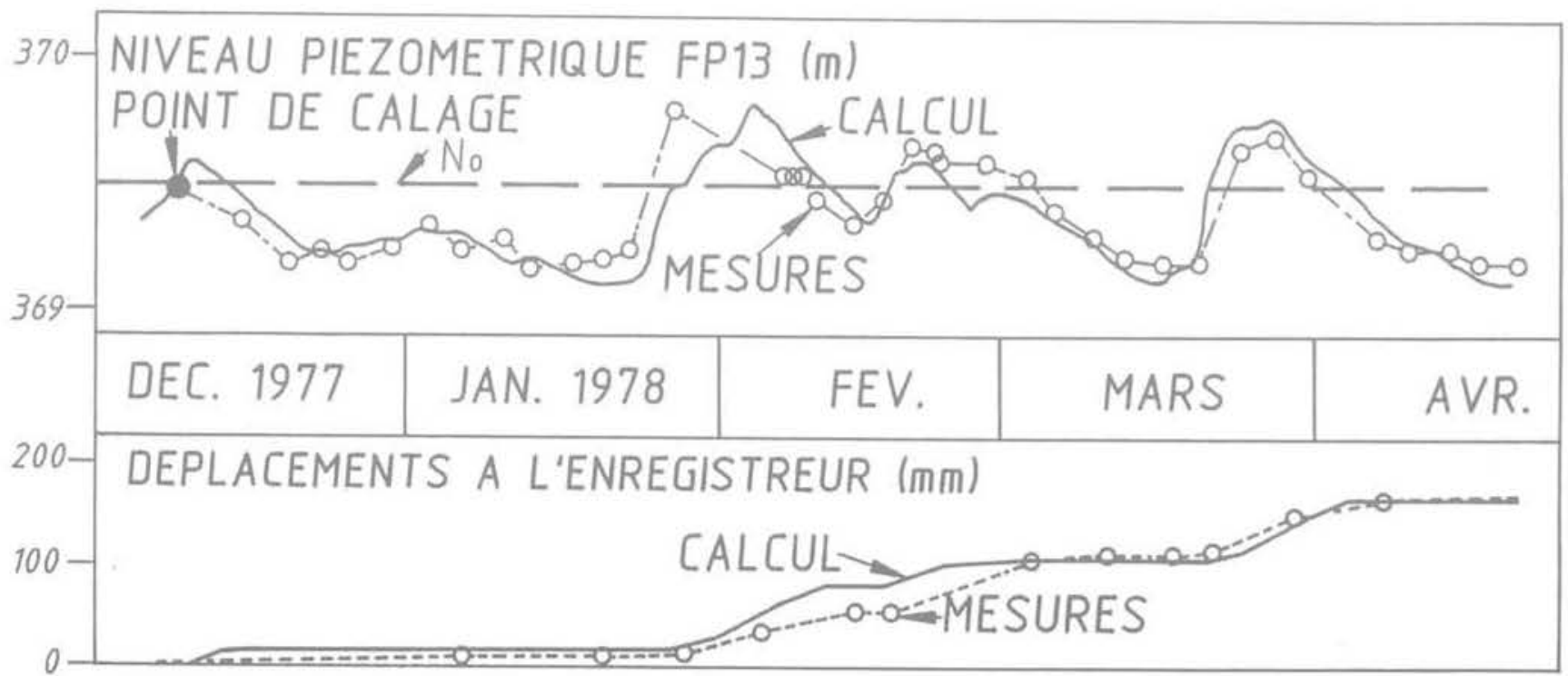

FG.5 Glissement de Leaz - Comparaison des niveaux piézométriques et des déplacements calculés à partir de la pluviométrie, avec les mêmes valeurs mesurées.

Leaz landslide - Comparison of calculated and measured piezometric level and displacements. 
le résultat de l'application de ces modèles et on peut en conclure que, tant que ce mécanisme reste identique, pour les séquences pluviométriques connues, les vitesses pourront atteindre environ $10 \mathrm{~mm} / \mathrm{j}$ puis diminueront.

Par conséquent dans ce type de mouvement, ni les mouvements, ni l'accélération seuls sont des critères suffisants pour déterminer le moment où le mouvement devient rapide et irréversible. Il faut considérer l'ensemble (sollicitations + déplacements) pour déterminer ce moment. Les critères de danger correspondent soit à une accélération sous contrainte constante, soit à des vitesses nettement supérieures (pour une même contrainte) à celles déterminées auparavant.

\section{4}

\section{Critique de ces modèles}

Le modèle hydraulique appliqué ici prend en compte l'évapotranspiration de manière simplifiée. D'autre part, il ne prend pas en compte le stockage des précipitations sous forme de neige. Néanmoins, dans le cas de Leaz, nous le considérons comme acceptable.

Le modèle mécanique est simplifié, car il ne prend en compte que le niveau d'un seul piézomètre situé en partie basse de la zone active (Fig. 3). En fait la réponse cinématique au niveau de l'enregistreur de la zone active est non seulement fonction du niveau piézométrique ( global » mais aussi des poussées exercées par la partie amont et des butées éventuelles de parties aval, car le glissement ne se déplace pas en bloc.

\section{5}

\section{Applications à d'autres cas de glissements}

Les glissements de terrains dans des formations d'argiles glacio-lacustres sont nombreux au Sud de Grenoble dans le Trièves. Il était donc intéressant de chercher à leur appliquer la même méthodologie de surveillance qu'à Leaz. Cependant, si on a pu disposer de mesures de pluviométrie journalière à proximité, ainsi que de mesures piézométriques très fréquentes, les mesures de déplacements ont été beaucoup plus espacées. On n'a donc pas pu établir de corrélations entre vitesses et niveaux piézométriques, mais on a cherché à appliquer un modèle hydraulique analogue à celui de Leaz.

Les caractéristiques de ces glissements, en comparaison de celles de Leaz sont les suivantes:

\begin{tabular}{|c|c|c|c|}
\hline & ST-GULLAUME & PONSONNAS & LFAZ \\
\hline $\begin{array}{ll}\text { Argiles } & \mathrm{W}_{\perp} \% \\
& \mathrm{IP} \\
& \emptyset^{\prime} \mathrm{r}\left({ }^{\circ}\right)\end{array}$ & $\begin{array}{l}35-45 \\
19-27\end{array}$ & $\begin{array}{l}32-38 \\
13-21 \\
18-21\end{array}$ & $\begin{array}{c}35-50 \\
20-30 \\
19,5\end{array}$ \\
\hline Pente $\left({ }^{\circ}\right)$ & 8-12 & 8-18 & 12 \\
\hline $\begin{array}{l}\text { Profondeur } \\
\text { de glissement }\end{array}$ & $20 \mathrm{~m}$ & $17-45 \mathrm{~m}$ & $12 \mathrm{~m}$ \\
\hline Volumes $\left(\mathrm{m}^{3}\right)$ & $5.10^{6}$ & $10.10^{\circ}$ & $1,4.10^{6}$ \\
\hline $\begin{array}{l}\text { Vitesses moyennes } \\
\text { (cm/an) }\end{array}$ & $2-3$ & $1-2$ & $\begin{array}{l}1970: 5-20 \\
1993: 1-2\end{array}$ \\
\hline
\end{tabular}

Dans le cas de Saint-Guillaume (Fig.6) le modèle hydraulique donne des résultats satisfaisants. Il permet même, moyennant une estimation du niveau critique ( $1,5 \mathrm{~m}$ de profondeur), de reconstituer les ordres de grandeur des déplacements annuels.

Dans le cas de Ponsonnas (Fig. 7), le modèle hydraulique ne permet pas un bon ajustement avec les niveaux piézométriques observés. Ceci montre qu'il existe, en plus de l'alimentation directe par la surface, une alimentation latérale provoquant une variation du niveau de base, de période annuelle et d'amplitude $2 \mathrm{~m}$ dans le cas présent.

Ceci permet de tirer deux conclusions:

- dans la constitution du niveau piézométrique il y a une part d'origine locale (infiltration directe des précipitations tombant sur le bassin versant local englobant le glissement) et une part d'origine lointaine. C'est la variation du rapport entre les deux qui provoque des réponses diverses à des précipitations identiques: - lorsque la part d'alimentation directe est importante (cas de Leaz et de Saint-Guillaume) un réseau de drainage superficiel peut être très efficace car il intercepte une partie non négligeable des précipitations avant qu'elles ne s'infiltrent plus en profondeur. Au contraire dans des cas comme Ponsonnas, où la part d'alimentation profonde est non négligeable, le seul drainage superficiel n'aura qu'une efficacité limitée sur les mouvements.

\section{3}

\section{Exemple de l'éboulement du CD 926}

\section{Localisation - Circonstances}

Il s'agit d'un éboulement rocheux situé dans la vallée de l'Arvan, à $5 \mathrm{~km}$ au sud-ouest de Saint-Jean-deMaurienne.

Fin 1975, suite à un éboulement de l'ordre $1000 \mathrm{~m}^{3}$ dans les gypses en contrebas de la route départementale (CD 926), deux fissures distantes de $50 \mathrm{~m}$ sont apparues dans la chaussée de cette route. Les questions qui se sont alors posées ont été les suivantes:

- l'affaissement observé sur la route est-il local?

-y a-t-il un danger pour la route et lequel?

\section{2}

\section{Description du site}

Pour la zone étudiée, le CD 926 se situe à $100 \mathrm{~m}$ audessus de l'Arvan, dont il est séparé par les falaises de gypses à $45-50^{\circ}$.

Les terrains de couverture sont constitués d'éboulis et de dépôts morainiques (silt sableux à blocs arrondis).

Dans le gypse, on a noté les trois familles de plans de discontinuité suivantes:

- les plans de stratification à pendage 50 à $60^{\circ}$ vers l'aval, donc défavorables :

- une grande fissure à pendage $73^{\circ}$ vers le SE. Cette dernière est ouverte et comporte des traces de mouvements anciens. Sa position a été représentée sur la coupe de la figure 8 ; 


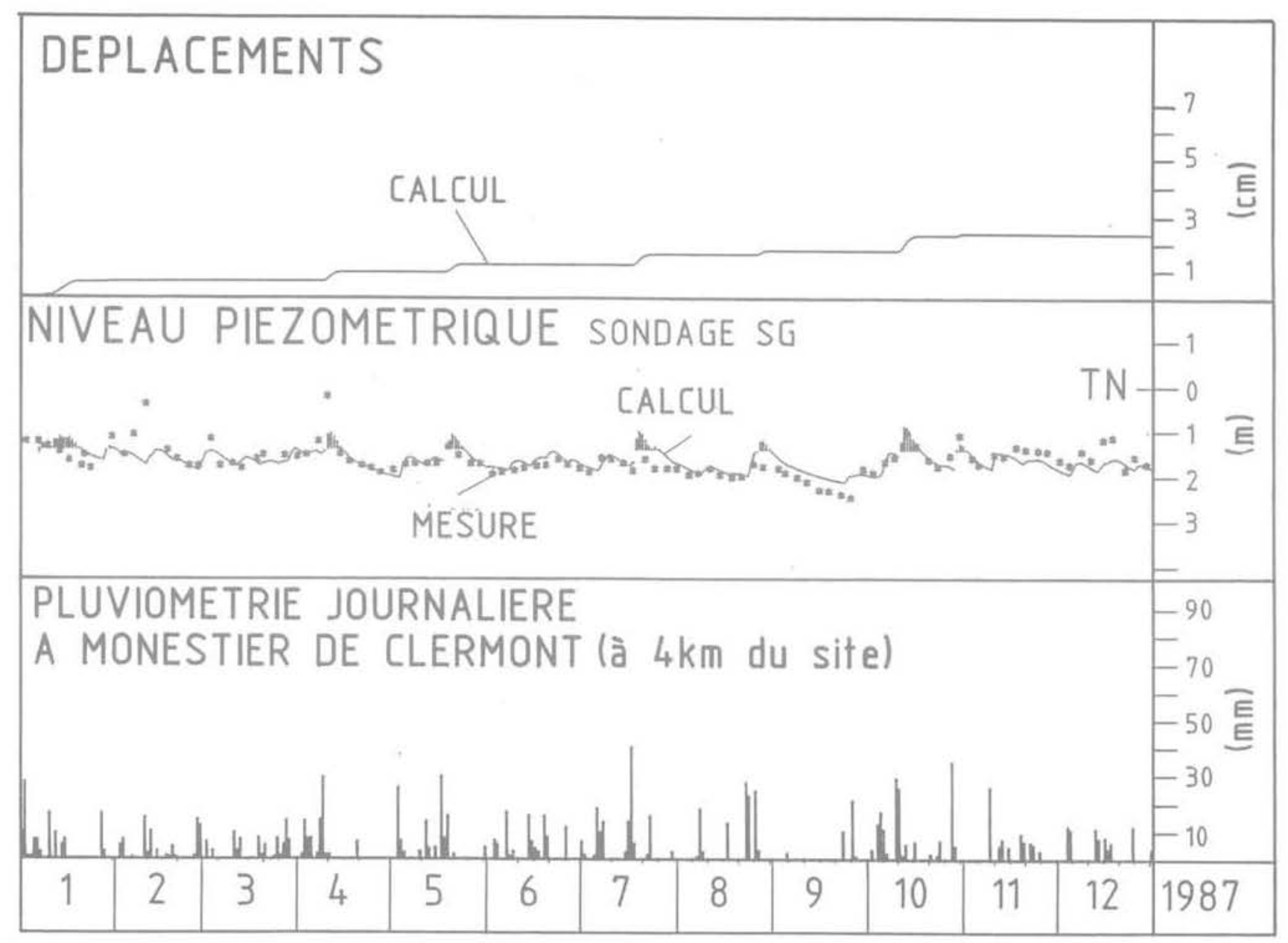

FG.6 Glissement de Saint-Guillaume (Isère) - Comparaison du niveau piézométrique calculé à partir de la pluviométrie à Monestier-de-Clermont (selon un modèle type Leaz), avec le niveau mesuré. Saint-Guillaume landslide - Comparison of calculated and measured piezometric level.

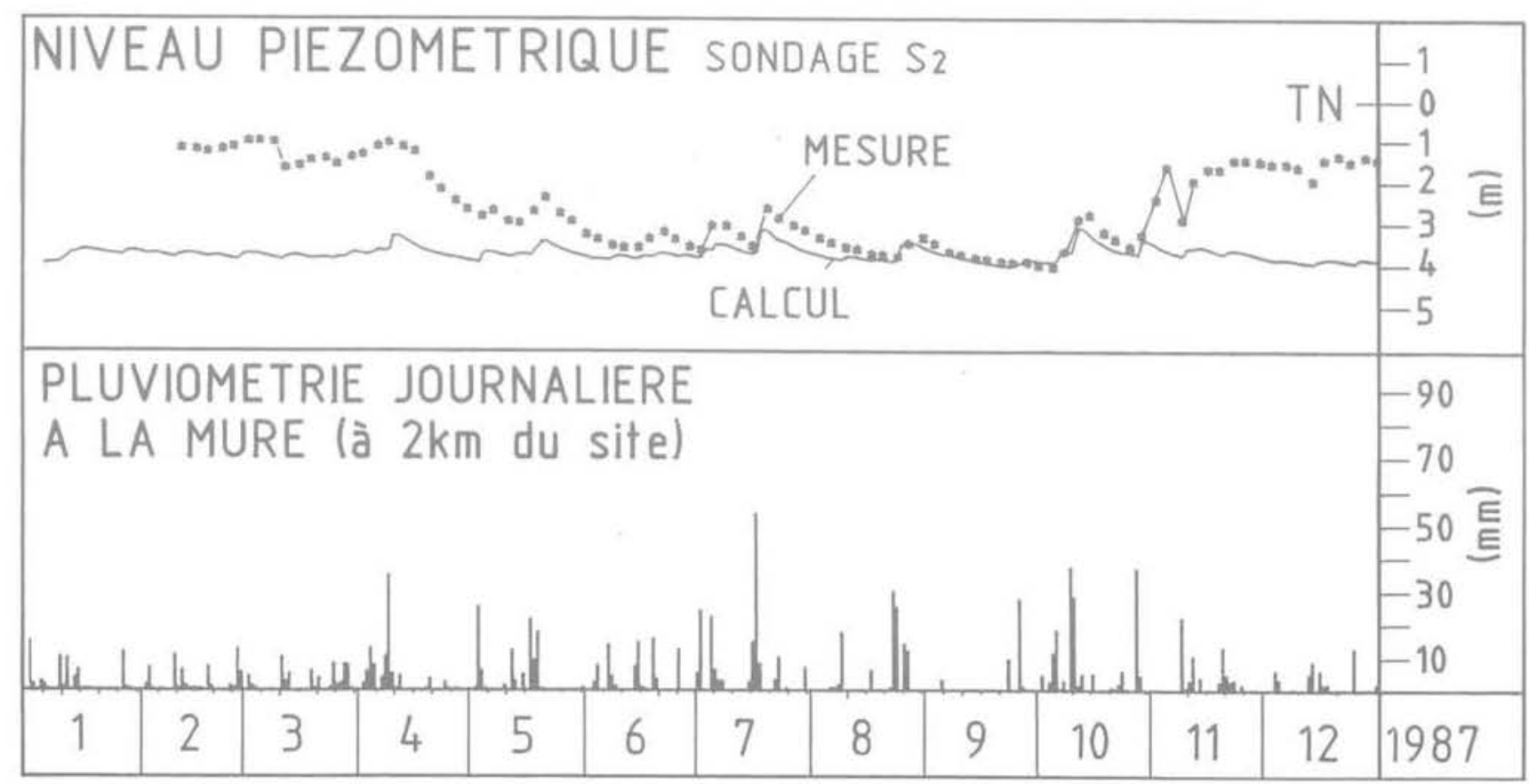

FIG.7 Glissement de Ponsonnas (Isère) -Comparaison du niveau piézométrique calculé à partir de la pluviométrie à La Mure (selon un modèle type Leaz), avec le niveau mesuré. 


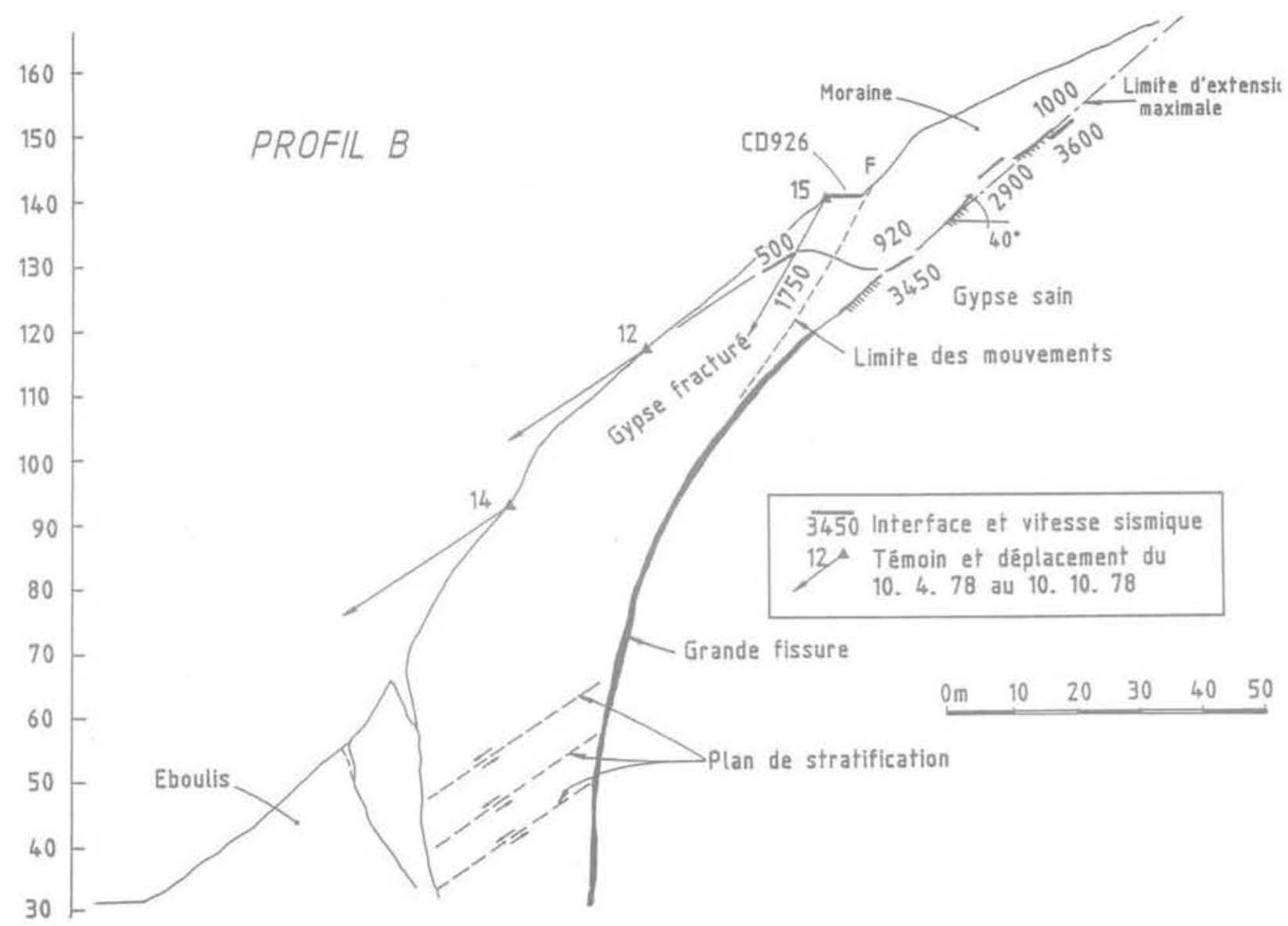

CD 926 - Profil en travers de l'éboulement.

CD 926 - Cross section of the rockslide.

- des plans de diaclases à pendage $60^{\circ}$ vers le SE, donc également défavorables.

Dans tous les terrains (substratum et couverture), on remarque l'absence de circulations d'eau importantes et de nappe.

\section{3}

\section{Surveillance}

En février 1976 , on a mis en place 10 repères de nivellement sur la route, puis en avril 1978, 7 repères dont 5 en contrebas de la route.

Dès le mois d'octobre 1978, on a pu faire les trois constatations suivantes:

- les vitesses de déplacements verticaux de témoins tels que 12 ou 14 étaient les mêmes que celles des repères de nivellement du CD 926, ce qui montrait bien qu'il y avait un mouvement d'ensemble (Fig. 8) ;

- les directions des vecteurs de déplacements des témoins 11 à 14 étaient parallèles entre elles, non conformes à la pente topographique, et cohérentes avec les directions et plongements des stries observées sur les plans de stratification ou de diaclases;

- les vitesses des mouvements étaient sensiblement constantes et non influencées par la pluviométríe (Fig. 9).

Mais, lors de la mesure du 11 février 1980, on a décelé une accélération, ce qui a conduit à resserrer les mesures (tous les 10 jours, puis tous les 2 jours, puis tous les jours). Au 5 mars 1980, l'analyse de l'évolution des déplacements a montré que la date la plus probable d'éboulement se situait le 8 ou 9 mars. On a recommandé alors la fermeture du CD 926 avec continuation de la surveillance. Au moment de la fermeture de la route (6 mars), les vitesses journalières d'affaissement ne dépassaient pas 5 à $7 \mathrm{~mm} /$ jour et l'accélération n'était pas décelable.

Le 8 mars à 21 h 30 , un éboulement estimé à $80000 \mathrm{~m}^{3}$ emportait le CD 926 sur $50 \mathrm{~m}$ de long. Les limites de cet éboulement ont été sensiblement celles qui avaient été prévues. Grâce à la fermeture préventive des routes, aucune victime n'a été à déplorer.

\section{4}

\section{Méthode de prévision}

Étant donné la mesure de l'évolution d'un paramètre significatif $X$ (déplacement, ou vitesse) en fonction du temps t, le problème se résume à la recherche d'une valeur finie $t_{r}$ pour laquelle $X$ tend vers l'infini.

Le principe, dérivé de la méthode proposée par Asaoka en 1978 pour le tassement cedométrique, consiste à découper les déplacements en intervalles égaux $\Delta D$ pour lesquels le temps successifs $t_{n}, t_{n+1}$ tendent vers une valeur $t_{r}$. Mais, ici, la relation $t_{n+1}=f\left(t_{n}\right)$ n'est pas obligatoirement linéaire. 


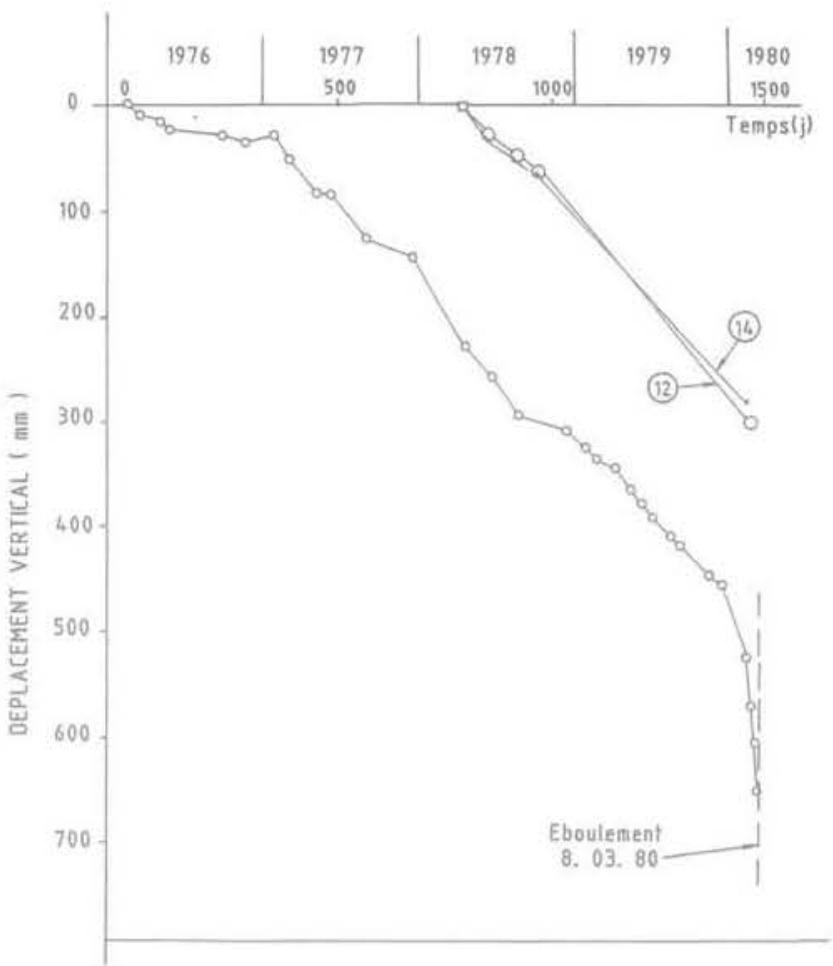

FIG:- CD 926 - Évolution des mouvements verticaux.

CD 926 - Vertical movements.

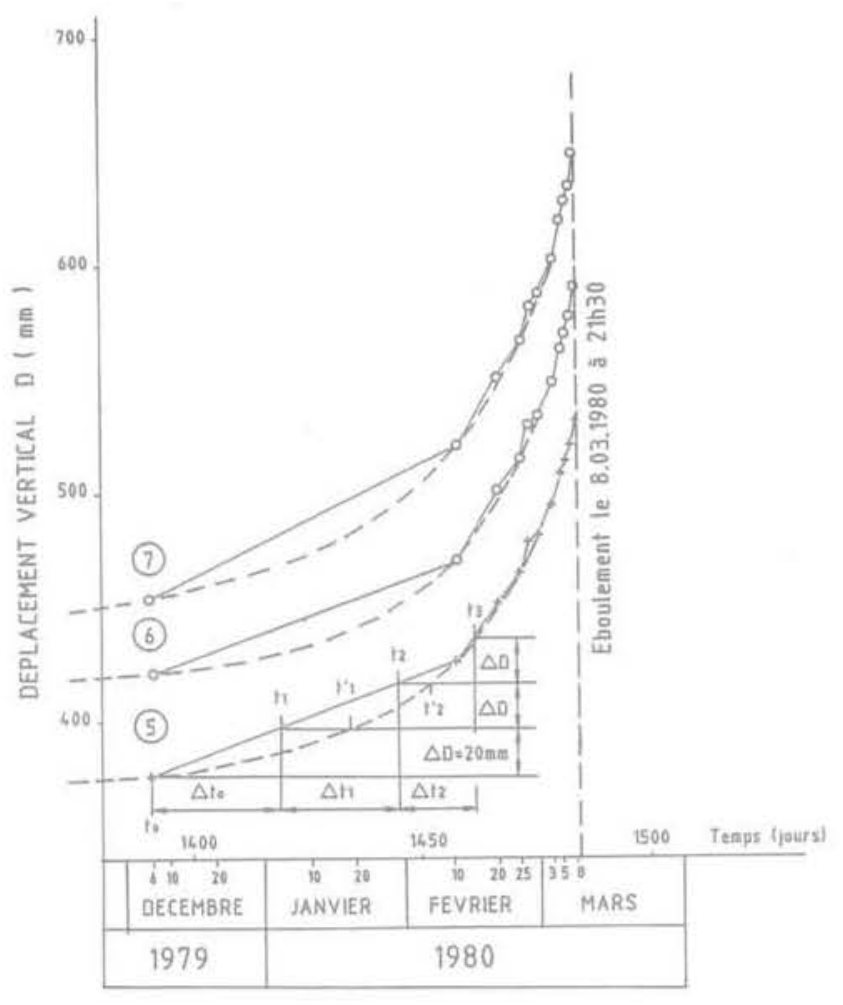

FG. 10 CD 926 - Courbe des mouvements verticaux durant la phase finale et principe du découpage des déplacements. CD 926 - Vertical movements in the ultimate stage, and principle of the forecasting method.
On a représenté sur la figure 10, pour 3 témoins: - les courbes brutes des déplacements verticaux mesurés au CD 926 dans les trois derniers mois (phase finale) ;

- les mêmes courbes, lissées:

- le principe du découpage des déplacements en intervalles successifs égaux permettant de définir la suite $t_{j}$, $t_{1}, t_{2} \ldots t_{n}$ sur les courbes brutes, ou $t_{0}, t^{\prime}, t_{2}^{\prime} \ldots t_{n}^{\prime}$ sur les courbes lissées.

L'intervalle de déplacement $\Delta \mathrm{D}$ est arbitraire. On a adopté, ici, $\Delta \mathrm{D}=20 \mathrm{~mm}$.

Sur la figure 11, on a représenté, pour les 3 témoins 5,6 et 7 , l'ensemble des points représentatifs $t_{n}=f\left(t_{n-1}\right)$ à partir des courbes lissées, en prenant $t_{0}=1391 \mathrm{j}$ (6 décembre 1979) correspondant au dernier point connu de la phase à vitesse sensiblement constante. Si, à une date donnée où l'on dispose d'un certain nombre de points, on essaie d'ajuster une droite par la méthode des moindres carrés et que l'on calcule l'intersection de celle-ci avec la bissectrice, on constate qu'on arrive à une date comprise entre le 8 et le 9/03/1980.

Si dans ce cas la prévision de la date de l'éboulement a pu être effectuée précisément c'est à cause de deux caractéristiques:

- le mouvement n'était aucunement influencé par les conditions climatiques et il s'agissait d'une rupture progressive dans le rocher;

- géométriquement, le mouvement ne pouvait se stabiliser en évoluant.

On va montrer dans les deux cas suivants, les difficultés posées si ces deux conditions ne sont pas remplies.

\section{4}

\section{Exemple de l'éboulement de Montvauthier}

\section{1}

\section{Localisation - Contexte géologique}

Ce cas concerne une falaise d'environ $40 \mathrm{~m}$ de haut sur la commune des Houches. Cette falaise est constituée de schistes gréseux et micacés du houiller comportant plusieurs directions de discontinuités dont au moins deux familles ont un pendage vers l'aval.

En contrebas, il existe une route et plusieurs habitations.

Les mesures sur 4 témoins ont commencé en juin 1992 sur des fissures qui sont apparues au sommet de la falaise à $5 \mathrm{~m}$ en arrière du bord de celle-ci.

Une prospection sismique par transparence a permis de préciser que le rocher était décomprimé sur 7 à $10 \mathrm{~m}$ de hauteur et 5 à $6 \mathrm{~m}$ d'épaisseur. Le volume en mouvement a été estimé à 800 ou $1000 \mathrm{~m}^{3}$.

\section{2}

\section{Surveillance - Travaux de protection}

La surveillance des mouvements a été réalisée par des mesures manuelles d'écartement des fissures avec une fréquence journalière. 


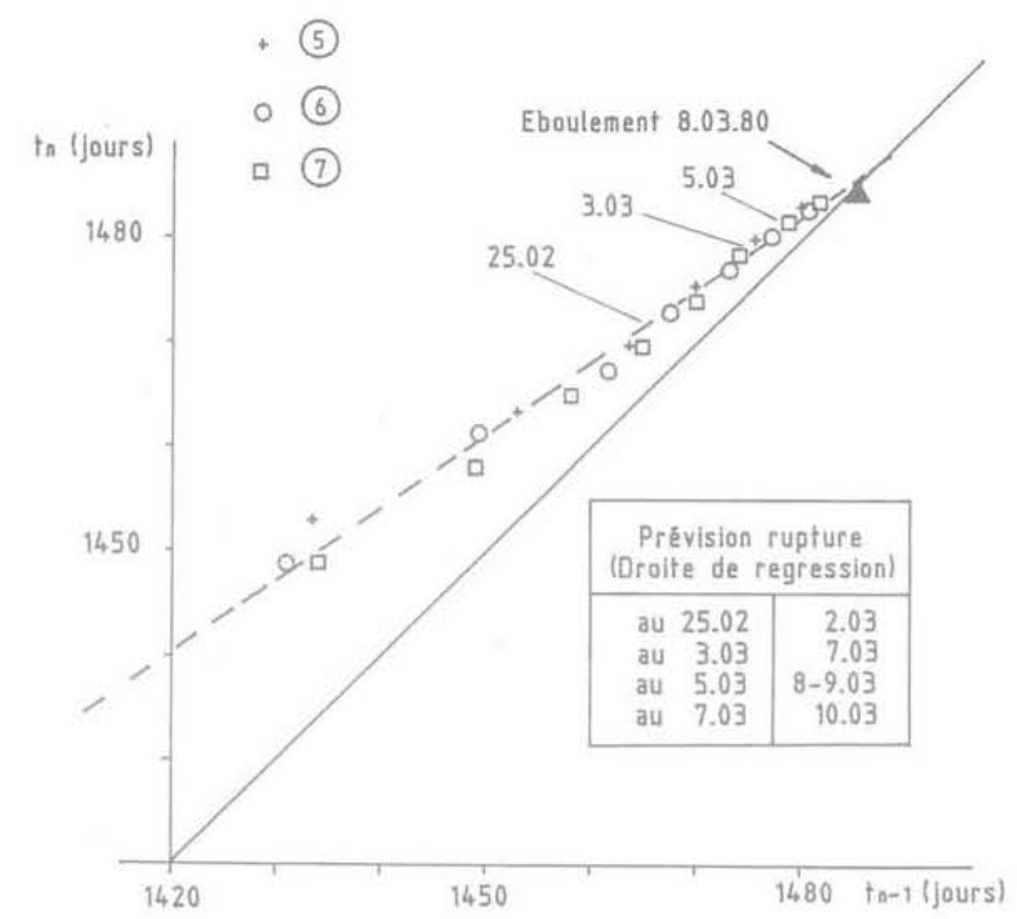

FIG, 11 CD $926-$ Relation $t_{n}=f\left(t_{n-1}\right)$ obtenue sur courbes de déplacements lissées, avec droite de régression au $5 / 03 / 1980$.

CD 926 - Function $t n=f(t n-1)$ obtained from the smoothed displacement curves, with regression curve on 05.03.1980.

L'appréciation des zones pouvant être atteintes par l'éboulement a été faite sur plusieurs profils selon la méthode de trajectographie de chutes de blocs mise au point par ADRGT. Il a été ainsi possible de vérifier que les maisons pouvaient être atteintes et de préconiser et dimensionner des merlons de protection qui ont été réalisês.

\section{Problème de la prévision de l'éboulement}

Sur la figure 12, on a reporté les courbes d'écartement des fissures et la pluviométrie journalière en fonction du temps. On peut constater que les mouvements s'accéléraient après des précipitations dépassant $20 \mathrm{~mm}$. Aucun niveau d'eau n'étant mesuré, il n'a pas été possible d'établir une corrélation entre les précipitations et les mouvements.

Ceci a été à l'origine d'une fausse alerte puisque l'analyse des mouvements au 6/09/1992 montrait une date de rupture probable (selon la méthode exposée au paragraphe 3) entre le 7 et le 9/09. Ceci était dû à l'accélération provoquée par les précipitations des 29 et 31/08.

Ce n'est qu'après le 9/09 que la courbe des déplacements a pris une allure régulière, sans ralentissement alors qu'il ne pleuvait pas. La rupture était amorcée, et la deuxième prévision effectuée le 17/09 indiquait une date de rupture la plus probable entre le 18 au soir et le 20 au matin. L'éboulement a eu lieu le 19 à $18 \mathrm{~h}$. Il avait été précédé par quelques chutes de blocs plusieurs heures auparavant.

Sur la figure 13, on a représenté schématiquement le corps de l'éboulement et les trajectoires des quelques blocs ayant été le plus loin. Ces dernières trajectoires correspondent bien à la prévision qui en avait été faite par les calculs trajectographiques. Au contraire le corps de l'éboulement a parcouru une faible distance, ayant été arrêté par la plate-forme constituée par la route.

Il est frappant de constater que le calcul trajectographique indiquait que $63 \%$ des blocs (donc une majorité) partis du sommet de falaise s'arrêtaient sur la route. Cet aspect qualitatif n'a pas été vérifié quantitativement puisqu'une infime partie de l'éboulement a atteint la zone occupée par les maisons. Ceci montre que la méthode de trajectographie pour le phénomène chutes de blocs ne peut être transposée d'une manière quantitative au phénomène éboulement.

\section{5}

\section{Exemple du glissement d'Auberives}

\section{1}

\section{Localisation - Contexte géologique}

Le glissement de terrain d'Auberives est situé en rive droite de la rivière la Bourne, affluent de l'Isère, à $40 \mathrm{~km}$ au sud-ouest de Grenoble. Il est très ancien et s'est réactivé deux fois récemment, en 1983 et en 


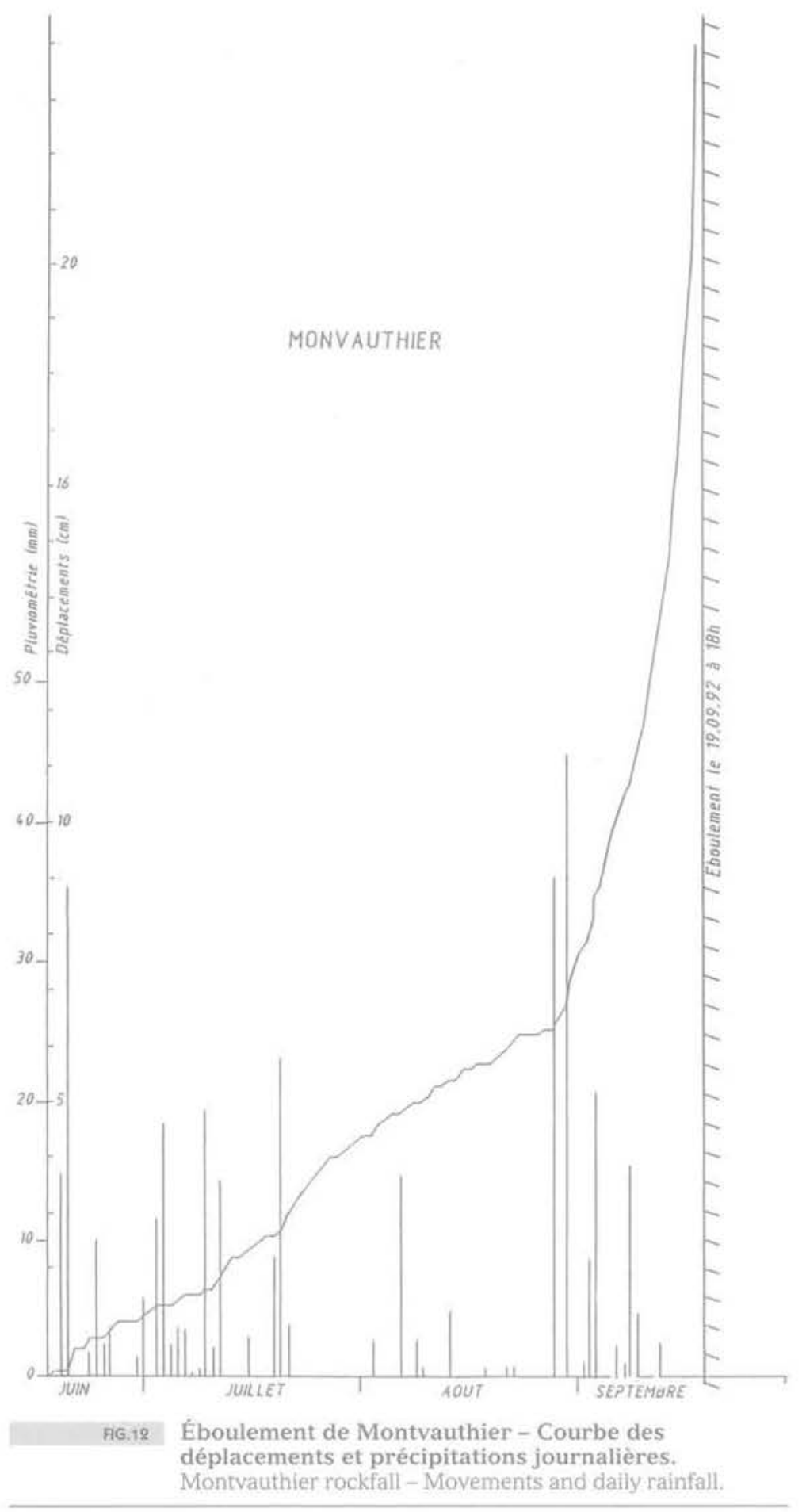

octobre 1988. Il affecte la route départementale CD 531 et, dans une moindre mesure, un canal d'irrigation (canal de la Bourne) qui a été implanté en tunnel à cet endroit dès 1875 à cause de ces mouvements.

La reprise des mouvements d'octobre 1988 s'est traduite par:

- une déformation du CD 531;

- une déformation du tunnel, beaucoup moins importante.

Le versant dominant la Bourne est haut de $120 \mathrm{~m}$, penté à $30-35^{\circ}$ en partie basse. La route se situe $15 \mathrm{~m}$ au-dessus de la Bourne. Les terrains sont composés de molasse : alternance de grès et marnes. Le pendage normal est dirigé vers $1^{\prime} E-S E$, de 5 à $15^{\circ}$.

Sur la figure 14 , on a représenté le profil en travers des glissements. Les mouvements détectés par les inclinomètres concernent:

- une partie superficielle de molasse sablo-gréseuse très décomprimée, épaisse de 5 à $10 \mathrm{~m}$, dans laquelle ont eu lieu les mouvements les plus actifs (A sur la figure 14);

- une zone profonde (B), descendant jusqu'à 15-35 m et affectée de mouvements lents, détectés dans le tunnel.

Dans la suite, on ne s'intéressera qu'aux mouvements les plus actifs. 


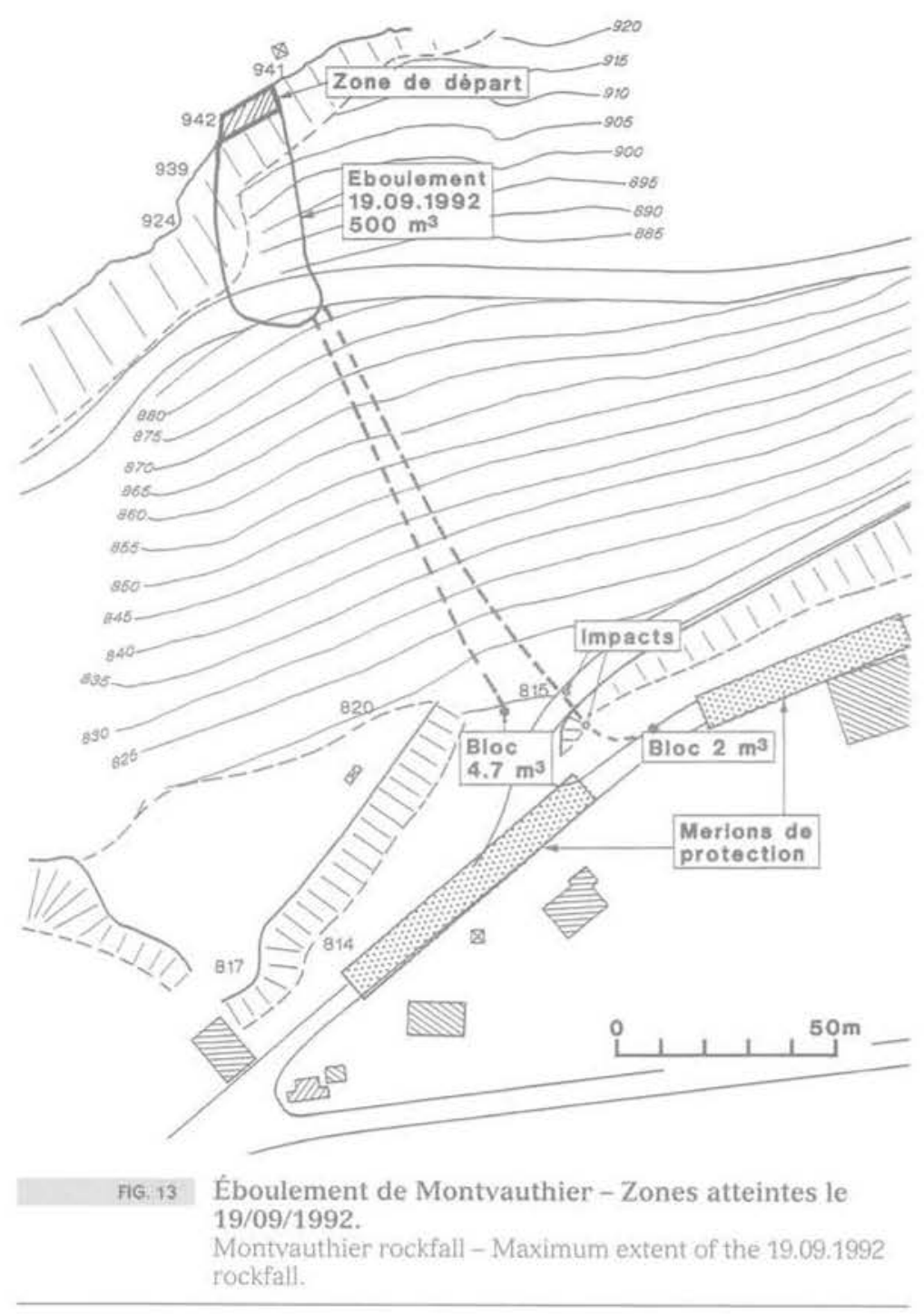

S

sF bis

PROFIL 1

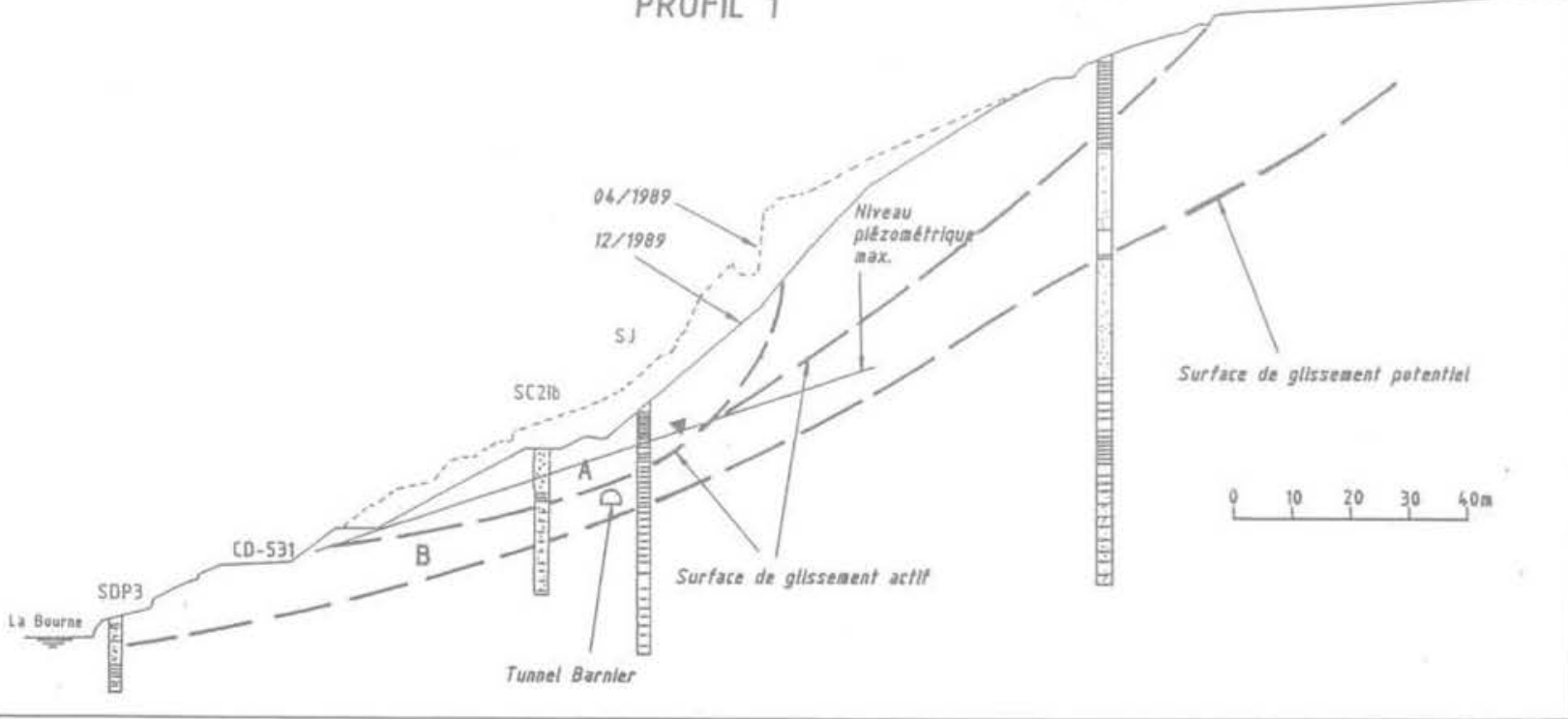

F10.14 Glissement d'Auberives - Profils en travers.

Auberives landslide - Geological cross section. 


\section{2}

\section{Problème de la prévision}

Lors de la reprise des mouvements en février 1989 , un certain nombre de repères de nivellement ont été implantés sur le CD 531 et relevés avec une fréquence de 5 fois/semaine. En février et mai, la courbe des affaissements a montré une nette accélération (Fig. 15), si bien qu'on a tenté de réaliser des prévisions sur les dates possibles d'éboulement. Cependant, dans ce cas, plus on avançait et plus les dates prévues reculaient.

$\begin{array}{ll}\text { Date de la prévision } & \begin{array}{l}\text { Date de la rupture } \\ \text { prévue }\end{array} \\ 18-03 & 25-03 \\ 21-03 & 26-03 \\ 22-03 & 27-03 \\ 23-03 & 30-03\end{array}$

Finalement, la vitesse est passée le 1er avril par un maximum de l'ordre de $7 \mathrm{~cm} / \mathrm{j}$ puis a ralenti. Ceci est dû à deux phénomènes:

- la modification de forme du glissement au cours des mouvements avec engraissement de la partie basse stabilisatrice et allégement de la partie amont motrice; - la dissipation des pressions interstitielles au fur et à mesure des mouvements.

\section{6}

\section{Conclusions}

Les exemples ci-dessus montrent que certains problèmes ont actuellement une solution possible même si elle est parfois lourde:

- pour les mouvements de terrains dont la vitesse est variable dans le temps, il est possible par mise en place d'une surveillance judicieuse (précipitations, déplacements et niveau piézométrique) d'établir des corrélations entre sollicitations et déplacements et de déterminer les particularités cinématiques des mouvements.

Il est alors possible de discerner le passage des mouvements à vitesses réversibles aux mouvements à vitesses irréversibles aboutissant à de grandes vitesses, si la loi de comportement définie expérimentalement d'après la surveillance n'est pas affectée par les déplacements (modification des conditions hydrauliques ou redistribution des masses);

- pour les petits éboulements et les chutes de blocs. quelques méthodes de calculs trajectographiques permettent de prévoir, avec une bonne approximation, les probabilités d'atteinte de diverses zones et également de dimensionner des protections efficaces (calcul de l'énergie et de la hauteur de passage);

- pour des menaces d'éboulements rocheux répondant à certaines conditions, on peut apprécier avec une assez bonne précision le moment du passage aux mouvements accélérés rapides. Au contraire, pour un grand nombre de mouvernents affectant des matériaux rocheux, cette dernière prévision est plutôt aléatoire (toutefois une augmentation de la fréquence des chutes de blocs constitue parfois un signe précurseur de grand éboulement).

Les difficultés dans ce dernier cas proviennent de plusieurs facteurs:

- les grandes masses rocheuses en mouvement ne peuvent pas souvent être considérées comme un système unique avec sa propre dynamique et doivent être subdivisées en plusieurs sous-ensembles, chacun d'eux pouvant avoir ses propres particularités dynamiques, d'où une complexité accrue du système de surveillance,

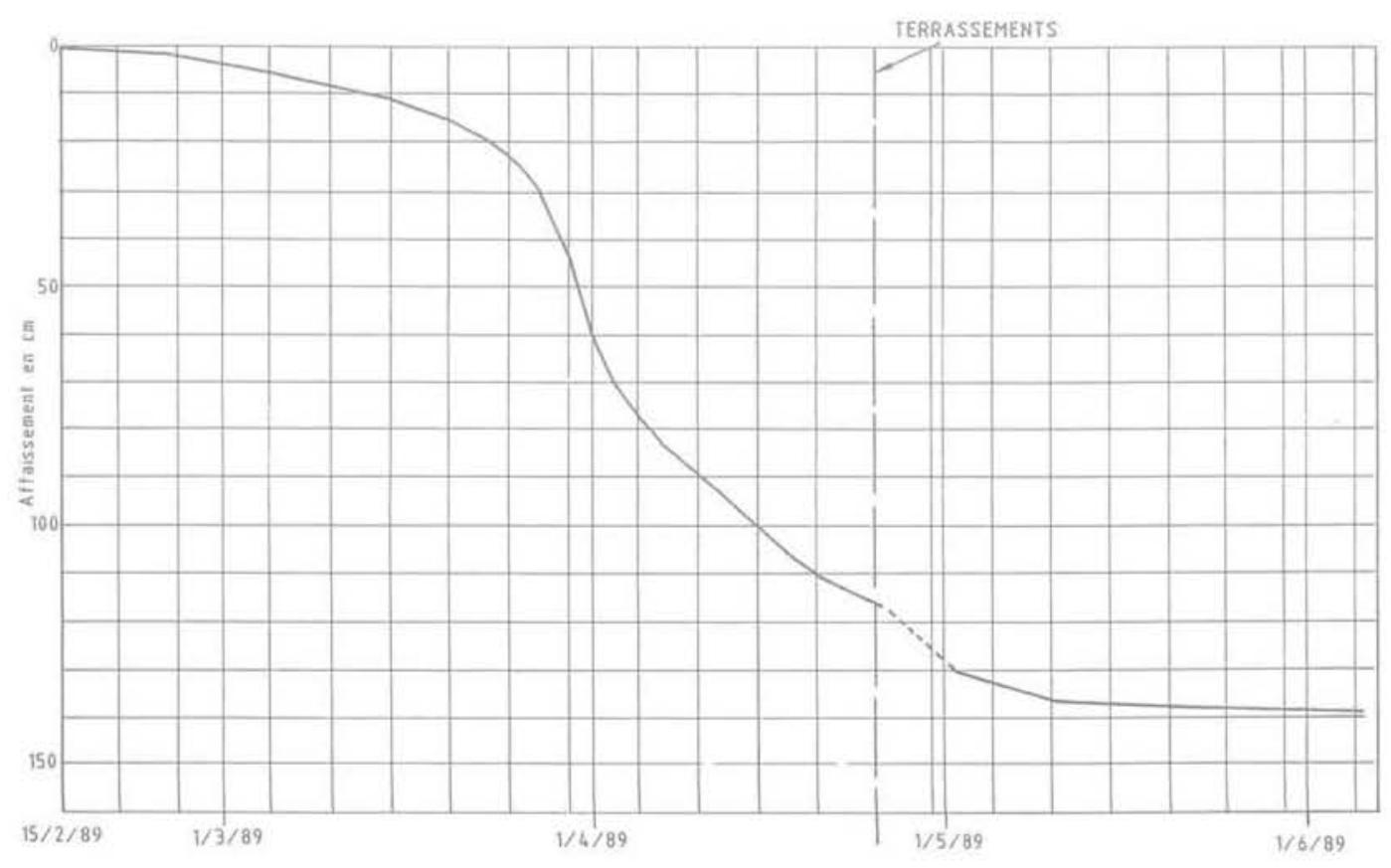

FIG. 15 Glissement d'Auberives - Courbe des mouvements verticaux du repère 125. Auberives landslide - Vertical movements at measuring point $n^{\circ} 125$. 
- les mesures sont difficiles à organiser s'il s'agit de falaises étendues,

- les corrélations entre pluviométrie et déplacements ne sont pas faciles à établir,

- on ne connaît pas, pour des matériaux rocheux, la durée de l'état (2) mais on peut supposer qu'elle est réduite. Ce manque d'expérience ne facilite pas l'organisation de la surveillance systématique (implantation des repères, espacement des mesures, interprétation).

\section{Bibliographie}

Asaoka A - Observational procedure of settlement prediction, Soils and Foundations 18 (4), 1978, p. $87-101$

Azími C., Desvarreux P., et al. - Méthode de calcul de la dynamique des chutes de blocs, Bull. Liaison Labo P. et Ch. 122, 1982, p. 93-102.

Azimi C., Biarez J., et al. - Prévision d'éboulement en terrain gypseux, Proc. 5 th internat. Svmp. on Landslides, Lausanne, 1988, p. 531-536.
Azimi C., Biarez J., et al. - Mécanisme des glissements de terrains argileux. Bilan de surveillance sur plusieurs années, Proc. 6th Internat. Symp. on Landslides, Christchurch, 1992, p. 1903-1908. 